\title{
GROWTH OF SOLUTIONS OF SECOND ORDER LINEAR DIFFERENTIAL EQUATIONS WITH EXTREMAL FUNCTIONS FOR DENJOY'S CONJECTURE AS COEFFICIENTS
}

\author{
JIANREN LONG
}

\begin{abstract}
The classical problem of finding conditions on the entire coefficients $A(z)$ and $B(z)$ guaranteeing that all nontrivial solutions of $f^{\prime \prime}+A(z) f^{\prime}+B(z) f=0$ are of infinite order is discussed. Some such conditions which involve deficient value, Borel exceptional value and extremal functions for Denjoy's conjecture are obtained.
\end{abstract}

\section{Introduction and main results}

The growth of solutions of complex linear differential equations is one of interesting topic in complex analysis after Wittich's work published [24]. It is studied by using Nevanlinna theory of meromorphic functions. Here we assume that the reader is familiar with the fundamental results and standard notation in Nevanlinna theory, see [12, 16, 28] for more details. In general, we use the order of growth to measure the growth of entire functions. For a function $f$ entire in the complex plane $\mathbb{C}$, the order of growth and lower order of growth are given by

$$
\rho(f)=\limsup _{r \rightarrow \infty} \frac{\log ^{+} \log ^{+} M(r, f)}{\log r}
$$

and

$$
\mu(f)=\liminf _{r \rightarrow \infty} \frac{\log ^{+} \log ^{+} M(r, f)}{\log r},
$$

respectively. In present paper, we will continuously mention this topic, considering the growth of solutions of the equation

$$
f^{\prime \prime}+A(z) f^{\prime}+B(z) f=0,
$$

Received May 8, 2015, accepted July 29, 2015.

2010 Mathematics Subject Classification. Primary 34M10; Secondary 30D35.

Key words and phrases. Complex differential equation, Borel exceptional value, infinite order, Denjoy's conjecture, deficient value. 
where $A(z)$ and $B(z) \not \equiv 0$ are entire functions. It is well known that if $A(z)$ and $B(z)$ are entire functions, then all solutions of (1.1) are entire functions, and if there exists at least one transcendental coefficient and $f_{1}, f_{2}$ are two linearly independent solutions of (1.1), then at least one of $f_{1}, f_{2}$ must have infinite order. Hence, "most" solutions of (1.1) have infinite order. On the other hand, there are equations of the form (1.1) that possess a nontrivial solution of finite order; for example, $f(z)=e^{z}$ satisfies $f^{\prime \prime}+e^{-z} f^{\prime}-\left(e^{-z}+1\right) f=0$. Thus a natural question is: What conditions on $A(z)$ and $B(z)$ will guarantee that every nontrivial solution of (1.1) is of infinite order? Many such results are obtained in the literature. The following theorem on this question is a summary of results due to Gundersen [10], Hellerstein, Miles and Rossi [11], and Ozawa [21].

Theorem A. Suppose that $A(z)$ and $B(z)$ are entire functions satisfying one of the following conditions.

(i) $\rho(A)<\rho(B)$;

(ii) $A(z)$ is polynomial and $B(z)$ is transcendental;

(iii) $\rho(B)<\rho(A) \leq \frac{1}{2}$.

Then every nontrivial solution of (1.1) is of infinite order.

By Theorem A, the main problem left to consider is that whether every nontrivial solution of (1.1) is of infinite order if $\rho(A)=\rho(B)$ or if $\rho(A)>\frac{1}{2}, \rho(B)<\rho(A)$. In general, the conclusions are false for these situations. For example, $f(z)=\exp (P(z))$ satisfies the equation

$$
f^{\prime \prime}+A(z) f^{\prime}+\left(-P^{\prime \prime}(z)-\left(P^{\prime}(z)\right)^{2}-A(z) P^{\prime}(z)\right) f=0,
$$

where $A(z)$ is an entire function and $P(z)$ is a nonconstant polynomial. For the case of $\rho(B)<$ $\rho(A)$, there are also some examples [10] showing that a nontrivial solution of (1.1) has finite order.

The problem of finding conditions on $A(z)$ and $B(z)$ for the case $\rho(A) \geq \rho(B)$ under which all nontrivial solutions of (1.1) are of infinite order has raised considerable interest, and many parallel results on this question written after Theorem A, see, for example, $[3,4,14,15,17$, 18, 22]. It would be interesting to get some relations between the growth of solutions of (1.1) and some deep results in Nevanlinna theory of meromorphic functions. It is well known that deficient value and Borel direction play fundamental role in Nevalinna theory of meromorphic functions and many interesting work done on this aspect, see, for example, [29]. Some relationships between the growth of solutions of (1.1) and deficient value of coefficients are found, see, for example, [15, 25]. Borel direction plays a basic role in the theory of angular distribution of meromorphic functions, lots of results can be found, see, for example, $[8,20,26,30,31]$. In [18], some relationships between the growth of solutions of (1.1) and angular distribution of coefficients are found. 
Now we consider equation (1.1) again by using some deep results in Nevanlinna theory. To this end, we begin by recalling a conjecture due to Denjoy [6] from 1907, verified by Ahlfors [1] in 1930 .

Denjoy's Conjecture. Let $f$ be an entire function of finite order $\rho$. If $f$ has $k$ distinct finite asymptotic values, then $k \leq 2 \rho$.

An entire function $f$ is called an extremal function for Denjoy's conjecture if it is of finite order $\rho$ and has $k=2 \rho$ distinct finite asymptotic values. These functions are investigated by Ahlfors [1], Drasin [7], Kennedy [13] and Zhang [32], to mention a few.

An example of an extremal function for Denjoy's conjecture is

$$
f(z)=\int_{0}^{z} \frac{\sin t^{q}}{t^{q}} d t
$$

where $q$ is a positive integer. Then $\rho(f)=q$, and $f$ has $2 q$ distinct finite asymptotic values

$$
a_{l}=e^{\frac{l \pi i}{q}} \int_{0}^{\infty} \frac{\sin r^{q}}{r^{q}} d r
$$

with its corresponding $2 q$ asymptotic curves being

$$
\arg z=\frac{l \pi}{q},
$$

where $l=1,2, \ldots, 2 q$, see [33, p. 210] for more details.

The first result shows the relationship between the fast growth of solutions of (1.1) and extremal functions for Denjoy's conjecture as coefficient.

Theorem 1.1. Let $A(z)$ be an entire function having a finite Borel exceptional value, and let $B(z)$ be an extremal function for Denjoy's conjecture. Then every nontrivial solution of (1.1) is of infinite order.

From Theorem 1.1, we can easily obtain the following corollary.

Corollary 1.2. Let $A(z)$ be an entire function having a finite Picard exceptional value, and let $B(z)$ be an extremal function for Denjoy's conjecture. Then every nontrivial solution of (1.1) is of infinite order.

The proofs of Theorem 1.1 and of the remaining theorems involve logarithmic densities of sets, we proceed to recall these concepts. The Lebesgue linear measure of a set $E \subset[0, \infty)$ is $\mathrm{m}(E)=\int_{E} d t$, and the logarithmic measure of a set $F \subset[1, \infty)$ is $\mathrm{m}_{1}(F)=\int_{F} \frac{d t}{t}$. The upper and lower logarithmic densities of $F \subset[1, \infty)$ are given, respectively, by

$$
\overline{\log \operatorname{dens}}(F)=\limsup _{r \rightarrow \infty} \frac{\mathrm{m}_{1}(F \cap[1, r])}{\log r}
$$


and

$$
\underline{\log \operatorname{dens}}(F)=\liminf _{r \rightarrow \infty} \frac{\mathrm{m}_{1}(F \cap[1, r])}{\log r}
$$

In order to state our results, we also need some definitions. For $\theta \in \mathbb{R}$, let $\Delta(\theta)=\left\{r e^{i \theta}: r \geq 0\right\}$. For $\alpha<\beta$ and $r_{1}, r_{2} \in(0, \infty)$, define

$$
\begin{aligned}
& S(\alpha, \beta)=\{z:|z|>0, \alpha<\arg z<\beta\}, \\
& S\left(\alpha, \beta ; r_{1}, r_{2}\right)=\left\{z: r_{1}<|z|<r_{2}, \alpha<\arg z<\beta\right\} .
\end{aligned}
$$

Now, it is natural to ask the following question: What can we say if finite Borel exceptional value is replaced with finite deficient value in Theorem 1.1? We begin with recalling a result concerning the relationship between growth of solutions of (1.1) and deficient value of coefficients, which is due to $\mathrm{Wu}-\mathrm{Zhu}$ [25, Theorem 1.1].

Theorem B. Let $A(z)$ be an entire function having a finite deficient value, and let $B(z)$ be a transcendental entire function with $\mu(B)<\frac{1}{2}$. Then every nontrivial solution of $(1.1)$ is of infinite order.

Here we obtain an analogue of Theorem B in which the assumption $\mu(B)<\frac{1}{2}$ is replaced with extremal functions for Denjoy's conjecture, and an additional condition of angular distribution is needed.

Theorem 1.3. Let $A(z)$ be an entire function having a finite deficient value, and let $B(z)$ be an extremal function for Denjoy's conjecture and $\mathrm{m}\left(E_{0}\right)=0$, where $E_{0}=\{\theta \in[0,2 \pi]: \Delta(\theta)$ is Borel direction of $B(z)\}$. Then every nontrivial solution of (1.1) is of infinite order.

In fact, $B(z)$ has at least one finite asymptotic value by the assumption of Theorem 1.3, hence $\mu(B) \geq \frac{1}{2}$ by using [33, Theorem 4.4]. Applying [33, Corollary 1, p. 226] and the similar reasoning in proving Theorem 1.3, we have immediately the following result in which Julia direction is considered, we omit the details of the proof.

Theorem 1.4. Let $A(z)$ be an entire function having a finite deficient value, and let $B(z)$ be an extremal function for Denjoy's conjecture and $\mathrm{m}\left(E_{0}\right)=0$, where $E_{0}=\{\theta \in[0,2 \pi]: \Delta(\theta)$ is Julia direction of $B(z)\}$. Then every nontrivial solution of (1.1) is of infinite order.

\section{Auxiliary results}

We begin with recalling a lemma on logarithmic derivatives due to Gundersen [9], which plays an important role in proving our results. 
Lemma 2.1. Let $f$ be a transcendental meromorphic function of finite order $\rho(f)$. Let $\varepsilon>0$ be given real constant, and let $k$ and $j$ be integers such that $k>j \geq 0$. Then there exists a set $E \subset[0,2 \pi)$ with $\mathrm{m}(E)=0$, such that if $\psi_{0} \in[0,2 \pi)-E$, then there is a constant $R_{0}=R_{0}\left(\psi_{0}\right)>1$ such that for all $z$ satisfying $\arg z=\psi_{0}$ and $|z| \geq R_{0}$, we have

$$
\left|\frac{f^{(k)}(z)}{f^{(j)}(z)}\right| \leq|z|^{(k-j)(\rho(f)-1+\varepsilon)} .
$$

We proceed to recall a property of extremal functions for Denjoy's conjecture, which is needed in proving our results.

Lemma 2.2 ([33, Theorem 4.11]). Let $f$ be an extremal function for Denjoy's conjecture. Then, for any $\theta \in[0,2 \pi)$, either $\Delta(\theta)$ is a Borel direction of $f$, or there exists a constant $\sigma \in\left(0, \frac{\pi}{4}\right)$, such that

$$
\lim _{\substack{|z| \rightarrow \infty \\ z \in(S(\theta-\sigma, \theta+\sigma)-E)}} \frac{\log \log |f(z)|}{\log |z|}=\rho(f),
$$

where $E$ denotes a subset of $S(\theta-\sigma, \theta+\sigma)$, and satisfies

$$
\lim _{r \rightarrow \infty} \mathrm{m}(S(\theta-\sigma, \theta+\sigma ; r, \infty) \cap E)=0 .
$$

The next lemma is related with Borel exceptional value.

Lemma 2.3 ([27, Theorem 2.11]). Let $f$ be a meromorphic function of order $\rho(f)>0$. If $f$ has two distinct Borel exceptional values, then $f$ is of regular growth and its (lower) order is a positive integer or $\infty$.

The following lemma due to Markushevich [19], see also [2, Lemma 2.3].

Lemma 2.4. Let $P(z)=b_{n} z^{n}+b_{n-1} z^{n-1}+\cdots+b_{0}$, where $n$ is a positive integer and $b_{n}=\alpha_{n} e^{i \theta_{n}}$, $\alpha_{n}>0, \theta_{n} \in[0,2 \pi)$. For any given $\varepsilon \in\left(0, \frac{\pi}{4 n}\right)$, we introduce $2 n$ open angles

$$
S_{j}=\left\{z:-\frac{\theta_{n}}{n}+(2 j-1) \frac{\pi}{2 n}+\varepsilon<\arg z<-\frac{\theta_{n}}{n}+(2 j+1) \frac{\pi}{2 n}-\varepsilon\right\},
$$

where $j=0,1, \ldots, 2 n-1$. Then there exists a positive number $R=R(\varepsilon)$ such that for $|z|=r>R$,

$$
\operatorname{Re}\{P(z)\}>\alpha_{n}(1-\varepsilon) \sin (n \varepsilon) r^{n}
$$

if $z \in S_{j}$ when $j$ is even; while

$$
\operatorname{Re}\{P(z)\}<-\alpha_{n}(1-\varepsilon) \sin (n \varepsilon) r^{n}
$$

if $z \in S_{j}$ when $j$ is odd. 
Now for any given $\arg z=\theta \in[0,2 \pi)$, if $\theta \neq-\frac{\theta_{n}}{n}+(2 j-1) \frac{\pi}{2 n}, j=0,1, \ldots, 2 n-1$, then we take $\varepsilon$ sufficiently small, there exists some $S_{j}$ such that $z \in S_{j}$, where $j \in\{0,1, \ldots, 2 n-1\}$.

Finally, we also need the following result from [23, Corollary 2.3.6]. The proof relies on the Phragmén-Lindelöf principle.

Lemma 2.5. Let $f$ be entire function of order $\rho(f) \in(0, \infty)$. Then there exists an angular domain $S(\alpha, \beta)$ with $\beta-\alpha \geq \frac{\pi}{\rho(f)}$, where $\alpha$ and $\beta$ are constants, such that

$$
\limsup _{r \rightarrow \infty} \frac{\log \log \left|f\left(r e^{i \theta}\right)\right|}{\log r}=\rho(f)
$$

for all $\theta \in(\alpha, \beta)$.

We say that a half straight line $L_{\theta}: \arg z=\theta$ from the origin is called a radial line of order $\rho(f)$ of $f$ if $f$ satisfies (2.3), and an angular domain $S(\alpha, \beta)$ is called the radial angular domain of order $\rho(f)$ of $f$ if for every $\theta \in(\alpha, \beta), L_{\theta}$ is a radial line of order $\rho(f)$ of $f$. The two concepts can be found in [5]. There exists at least one the radial angular domain of order $\rho(f)$ for any entire function $f$ of order $\rho(f) \in(0, \infty)$ by Lemma 2.5 .

\section{Proof of Theorem 1.1}

If $\rho(A)=\infty$, then it is clear that $\rho(f)=\infty$ for every nontrivial solution $f$ of (1.1). Hence we may assume $\rho(A)<\infty$. Suppose on the contrary to the assertion that there is a nontrivial solution $f$ of $(1.1)$ with $\rho(f)<\infty$. We aim for a contradiction. By our assumption, suppose that $a$ is a finite Borel exceptional value of $A(z)$. Set

$$
g(z)=A(z)-a
$$

Then $g(z)$ has two Borel exceptional values 0 and $\infty$. Applying Lemma 2.3 and Hadamard's factorization theory, $g(z)$ takes the form

$$
g(z)=h(z) e^{Q(z)}
$$

where $h(z)$ is an entire function satisfying

$$
\rho(h)=\lambda(g)<\rho(g)
$$

and $Q(z)$ is a polynomial satisfying

$$
\operatorname{deg}(Q)=\rho(g)=\rho(A),
$$


where $\lambda(g)$ denotes the exponent of convergence of zeros of $g(z)$. Therefore,

$$
A(z)=h(z) e^{Q(z)}+a
$$

Let $Q(z)=b_{d} z^{d}+b_{d-1} z^{d-1}+\cdots+b_{0}$, where $b_{d}=\alpha_{d} e^{i \theta_{d}}, \alpha_{d}>0, \theta_{d} \in[0,2 \pi)$. For any given $\varepsilon \in\left(0, \min \left(\frac{\pi}{8 d}, \frac{\rho(B)}{2}\right)\right)$, let

$$
S_{j}=\left\{z:-\frac{\theta_{d}}{d}+(2 j-1) \frac{\pi}{2 d}+\varepsilon<\arg z<-\frac{\theta_{d}}{d}+(2 j+1) \frac{\pi}{2 d}-\varepsilon\right\},
$$

where $j=0,1, \ldots, 2 d-1$. By Lemma 2.4 and $\rho(h)<d=\rho(A)$, for any $\arg z=\theta \in\left(-\frac{\theta_{d}}{d}+(2 j-\right.$ 1) $\left.\frac{\pi}{2 d}+\varepsilon,-\frac{\theta_{d}}{d}+(2 j+1) \frac{\pi}{2 d}-\varepsilon\right)$ and $j$ is even,

$$
\left|A\left(r e^{i \theta}\right)-a\right|>\exp \left(C r^{d}\right)
$$

for all sufficiently large $r$, while for any $\arg z=\theta \in\left(-\frac{\theta_{d}}{d}+(2 j-1) \frac{\pi}{2 d}+\varepsilon,-\frac{\theta_{d}}{d}+(2 j+1) \frac{\pi}{2 d}-\varepsilon\right)$ and $j$ is odd,

$$
\left|A\left(r e^{i \theta}\right)-a\right|<\exp \left(-C r^{d}\right)
$$

for all sufficiently large $r$, where $C$ is a positive constant. We consider one of the $n$ sectors $S_{i}, i=1,3, \ldots, 2 d-1$. Without loss of generality, say $S_{1}=S\left(\alpha_{1}, \beta_{1}\right)$. This implies (3.2) holds for all $z=r e^{i \theta} \in S\left(\alpha_{1}, \beta_{1}\right)$ and enough large $r$. Next we consider the two cases appearing in Lemma 2.2.

(1) Suppose that the ray $\arg z=\theta$ is not Borel direction of $B(z)$, where $\alpha_{1}<\theta<\beta_{1}$. By Lemma 2.2, there exist a constant $\sigma \in\left(0, \frac{\pi}{8 d}\right)$ such that

$$
\lim _{\substack{|z| \rightarrow \infty \\ z \in\left(S(\theta-\sigma, \theta+\sigma)-E_{1}\right)}} \frac{\log \log |B(z)|}{\log |z|}=\rho(B),
$$

where $E_{1}$ denotes a subset of $S(\theta-\sigma, \theta+\sigma)$, and satisfies

$$
\lim _{r \rightarrow \infty} \mathrm{m}\left(S(\theta-\sigma, \theta+\sigma ; r, \infty) \cap E_{1}\right)=0
$$

Applying Lemma 2.1, there exists a set $E_{2} \subset[0,2 \pi)$ that has linear measure zero, such that if $\psi_{0} \in[0,2 \pi)-E_{2}$, then there is a constant $R_{0}=R_{0}\left(\psi_{0}\right)>1$ such that for all $z$ satisfying $\arg z=\psi_{0}$ and $|z| \geq R_{0}$, we have

$$
\left|\frac{f^{(k)}(z)}{f(z)}\right| \leq|z|^{2 \rho(f)}, \quad k=1,2 .
$$


Let $\Delta=\left\{z: \arg z=\psi, \psi \in E_{2}\right\}$. Then there exists a sequence of points $z_{n}$ with $z_{n} \rightarrow \infty$ as $n \rightarrow \infty,\left\{z_{n}\right\} \subset\left(S(\theta-\sigma, \theta+\sigma)-E_{1}\right) \cap\left(S_{1}-\Delta\right)$, such that

$$
\begin{aligned}
& \lim _{n \rightarrow \infty} \frac{\log \log \left|B\left(z_{n}\right)\right|}{\log \left|z_{n}\right|}=\rho(B), \\
& \left|A\left(z_{n}\right)-a\right|<\exp \left(-C\left|z_{n}\right|^{d}\right)
\end{aligned}
$$

and

$$
\left|\frac{f^{(k)}\left(z_{n}\right)}{f\left(z_{n}\right)}\right| \leq\left|z_{n}\right|^{2 \rho(f)}, \quad k=1,2 .
$$

By (1.1), we get

$$
|B(z)| \leq\left|\frac{f^{\prime \prime}(z)}{f(z)}\right|+|A(z)|\left|\frac{f^{\prime}(z)}{f(z)}\right| .
$$

Combining (3.4), (3.5), (3.6) and (3.7), we arrive at a contradiction for sufficiently large $n$. Thus, we have $\rho(f)=\infty$ for every nontrivial solution of (1.1).

(2) Suppose that the ray $\arg z=\theta$ is Borel direction of $B(z)$, where $\alpha_{1}<\theta<\beta_{1}$. Since $B(z)$ is extremal function for Denjoy conjecture, so $\rho(B) \geq \frac{1}{2}$ by using [33, Theorem 4.4]. Hence by using Lemma 2.5, there exists an angular domain $S(\alpha, \beta)$ with $\beta-\alpha \geq \frac{\pi}{\rho(B)}$, such that

$$
\limsup _{r \rightarrow \infty} \frac{\log \log \left|B\left(r e^{i \varphi}\right)\right|}{\log r}=\rho(B)
$$

for any $\alpha<\varphi<\beta$, where $0 \leq \alpha<\beta \leq 2 \pi$.

Let $S$ denotes the radial angular domain of order $\rho(B)$ of $B(z)$. Then, it follows from [5] that Borel direction of $B(z)$ either lie inside of $S$ or lie boundary of $S$. Obviously, $S(\alpha, \beta)$ is a radial angular domain of order $\rho(B)$ of $B(z)$. Hence, if $\arg z=\theta$ is boundary of $S(\alpha, \beta)$, without loss of generality, say $\theta=\beta$, then there exists a constant $\delta>0$ such that $S(\theta-\delta, \theta) \subset S(\alpha, \theta) \cap$ $S\left(\alpha_{1}, \beta_{1}\right)$, and (3.8) holds for any $\theta-\delta<\varphi<\theta$.

Applying Lemma 2.1, there exists $\varphi_{0} \in(\theta-\delta, \theta)$ and $R=R\left(\varphi_{0}\right)>1$, such that (3.3) holds for all $r>R$. Note that (3.2) holds for $\arg z=\varphi_{0}$, and

$$
\limsup _{r \rightarrow \infty} \frac{\log \log \left|B\left(r e^{i \varphi_{0}}\right)\right|}{\log r}=\rho(B) .
$$

Thus there is a sequence $r_{n}$ with $r_{n} \rightarrow \infty$ as $n \rightarrow \infty$, such that (3.2) and (3.3) hold for $|z|=r=$ $r_{n}$, and

$$
\left|B\left(r_{n} e^{i \varphi_{0}}\right)\right| \geq \exp \left(r_{n}^{\rho(B)-\varepsilon}\right)
$$


Therefore, we deduce from (3.2), (3.3), (3.7) and (3.9) that

$$
\begin{aligned}
& \exp \left(r_{n}^{\rho(B)-\varepsilon}\right) \leq\left|B\left(r_{n} e^{i \varphi_{0}}\right)\right| \\
& \leq\left|\frac{f^{\prime \prime}\left(r_{n} e^{i \varphi_{0}}\right)}{f\left(r_{n} e^{i \varphi_{0}}\right)}\right|+\left|\frac{f^{\prime}\left(r_{n} e^{i \varphi_{0}}\right)}{f\left(r_{n} e^{i \varphi_{0}}\right)}\right|\left(\left|A\left(r_{n} e^{i \varphi_{0}}\right)-a\right|+|a|\right) \\
& \leq r_{n}^{2 \rho(f)}\left(1+|a|+\exp \left(-C r_{n}^{d}\right)\right) .
\end{aligned}
$$

This is a contradiction for all sufficiently large $n$. Therefore Theorem 1.1 holds for the case $\arg z=\theta$ is boundary of $S(\alpha, \beta)$.

If $\arg z=\theta$ lie inside of $S(\alpha, \beta)$, then there exists a constant $\delta>0$ such that $S(\theta-\delta, \theta+\delta) \subset$ $S(\alpha, \beta) \cap S\left(\alpha_{1}, \beta_{1}\right)$. By using similar reasoning used in the case $\arg z=\theta$ is boundary of $S(\alpha, \beta)$, we get a contradiction. This completes the proof.

\section{Proof of Theorem 1.3}

As reasoning of the proof of Theorem 1.1, we may assume $\rho(A)<\infty$. Assume on the contrary to the assertion that there is a nontrivial solution $f$ of $(1.1)$ with $\rho(f)<\infty$. We aim for a contradiction. Suppose that $a$ is a finite deficient value of $A(z)$ with deficiency $\delta=\delta(a, A)$. By Lemma 2.1, there exists a set $E_{1} \subset[0,2 \pi)$ that has linear measure zero, such that if $\psi_{0} \in$ $[0,2 \pi)-E_{1}$, then there is a constant $R_{0}=R_{0}\left(\psi_{0}\right)>1$ such that (3.3) holds for all $z$ satisfying $\arg z=\psi_{0}$ and $|z| \geq R_{0}$.

By using similar way of [25, Lemma 2.5], there exists a sequence $r_{j}$ with $r_{j} \rightarrow \infty$ as $j \rightarrow \infty$, such that

$$
\mathrm{m}\left(E_{2}\right)=\mathrm{m}\left(\left\{\varphi \in[0,2 \pi):\left|A\left(r_{j} e^{i \varphi}\right)-a\right| \leq \exp \left(-\frac{\delta}{4} T\left(r_{j}, A\right)\right)\right\}\right) \geq d>0
$$

where $d$ is a constant depending on $\rho(A)$ and $\delta$. Applying Lemma 2.2, for any $\theta \in E_{2}-\left(E_{0} \cup E_{1}\right)$, there exists a constant $\sigma \in\left(0, \min \left(\frac{\pi}{4}, \frac{d}{4}\right)\right)$, such that

$$
\lim _{\substack{|z| \rightarrow+\infty \\ z \in\left(S(\theta-\sigma, \theta+\sigma)-E_{3}\right)}} \frac{\log \log |B(z)|}{\log |z|}=\rho(B),
$$

where $E_{3}$ denotes a subset of $S(\theta-\sigma, \theta+\sigma)$ satisfying

$$
\lim _{r \rightarrow \infty} \mathrm{m}\left(S(\theta-\sigma, \theta+\sigma ; r, \infty) \cap E_{3}\right)=0 .
$$

Let

$$
\Delta_{1}=\left\{z: \arg z=\theta \in E_{0} \cup E_{1}\right\}
$$


and

$$
\Delta_{2}=\left\{z: \arg z=\theta \in E_{2}\right\}
$$

Then there exists a sequence of point $z_{j}$ with $\left|z_{j}\right|=r_{j}$ (otherwise we use the subsequence $r_{j_{n}}$ instead of $\left.r_{j}\right)$ and $\left\{z_{j}\right\} \subset\left(\left(S(\theta-\sigma, \theta+\sigma)-E_{3}\right) \cap \Delta_{2}\right)-\Delta_{1}$, such that (3.3) and (4.1) hold for $|z|=r=r_{j}$, and

$$
\lim _{j \rightarrow \infty} \frac{\log \log \left|B\left(z_{j}\right)\right|}{\log \left|z_{j}\right|}=\rho(B) .
$$

Combining (3.3), (3.7), (4.1) and (4.2), we arrive at a contradiction as in the proof of Theorem 1.1. Thus, we have $\rho(f)=\infty$ for every nontrivial solution $f$ of (1.1). The proof is complete.

It is an open problem whether the conclusion of Theorem 1.3 still holds if the assumption $\mathrm{m}\left(E_{0}\right)=0$ is removed.

\section{Acknowledgements}

This research was partly supported by the Foundation of Science and Technology of Guizhou Province (Grant No. [2015]2112), the United Technology Foundation of Science and Technology Department of Guizhou Province and Guizhou Normal University (Grant No.LKS[2012] 12) China, the National Natural Science Foundation of China (Grant No. 11501142)

\section{References}

[1] L. Ahlfors, Untersuchungen zur Theorie der konformen abbildung und der Theorie der ganzen Funktionen, Acta Soc. Sci. Fenn., 1 (1930), 1-40.

[2] T. B. Cao, Growth of solutions of a class of complex differential equations, Ann. Polon. Math. 95 (2009), 141152.

[3] Z. X. Chen, The growth of solutions of $f^{\prime \prime}+e^{-z} f^{\prime}+Q(z) f=0$ where the order $(Q)=1$, Sci. China Ser. A, 45 (2002), 290-300.

[4] Z. X. Chen and C. C. Yang, Some further results on the zeros and growths of entire solutions of second order linear differential equations, Kodai Math. J., 22 (1999), 273-285.

[5] C. J. Dai and S. Y. Ji, Radial line of order $\rho$ and its relation to the distribution of Borel directions, J. Shanghai Normal Uni. Nat. Sci., 22 (1980), 16-24.

[6] A. Denjoy, A.Sur les fonctions entieres de genre fini, C. R. Acad. Sci. Paris, 45 (1907), 106-109.

[7] D. Drasin, On asymptotic curves of functions extremal for Denjoy's conjecture, Proc. London Math. Soc., 26 (1973), 142-166.

[8] D. Drasin and A. Weitsman, On the Julia Directions and Borel Directions of Entire Functions, Proc. London Math. Soc., 32 (1976), 199-212.

[9] G. G. Gundersen, Estimates for the logarithmic derivative of a meromorphic function, plus similar estimates, J. London Math. Soc., 37 (1988), 88-104.

[10] G. G. Gundersen, Finite order solution of second order linear differential equations, Trans. Amer. Math. Soc., 305 (1988), 415-429. 
[11] S. Hellerstein, J. Miles and J. Rossi, On the growth of solutions of $f^{\prime \prime}+g f^{\prime}+h f=0$, Trans, Amer. Math. Soc., 324 (1991), 693-705.

[12] W. K. Hayman, Meromorphic Functions, Clarendon Press, Oxford, 1964.

[13] P. B. Kennedy, A class of integral functions bounded on certain curves, Proc. London Math. Soc., 6 (1956), 518-547.

[14] K. Kwon, On the growth of entire functions satisfying second order linear differential equations, Bull. Korean Math. Soc., 33 (1996), 487-496.

[15] K. Kwon, K. and J. Kim, Maximum modulus, characteristic, deficiency and growth of solutions of second order linear differential equation, Kodai Math. J., 24 (2001), 344-351.

[16] I. Laine, Nevanlinna Theory and Complex Differential Equations, Walter de Gruyter Berlin, New York, 1993.

[17] I. Laine and P. C. Wu, Growth of solutions of second order linear differential equations, Proc. Amer. Math. Soc., 128 (2000), 2693-2703.

[18] J. R. Long, P. C. Wu and Z. Zhang, On the growth of solutions of second order linear differential equations with extremal coefficients, Acta Math. Sinica (English Series), 29 (2013), 365-372.

[19] A. I. Markushevich, Theory of Functions of a Complex Variable, Vol. П, Revised English Edition Translated and Edited by Richard A. Silverman. Prentice-Hall, Inc., Englewood Cliffs, N. J., 1965.

[20] M. Tsuji, On Borel's directions of meromorphic functions of finite order, Tohoku Math. J., 2 (1950), 97-112.

[21] M. Ozawa, On a solution of $w^{\prime \prime}+e^{-z} w^{\prime}+(a z+b) w=0$, Kodai Math. J., 3 (1980), 295-309.

[22] J. Wang and I. Laine, Growth of solutions of second order linear differential equations, J. Math. Anal. Appl., 342 (2008), 39-51.

[23] S. P. Wang, On the sectorial oscillation theory of $f^{\prime \prime}+A(z) f=0$, Ann. Acad. Sci. Fenn. Ser. A I Math. Diss., 92 (1994), 1-60.

[24] H. Wittich, Neuere untersuchungen über eindeutige analytische Funktionen, Springer-Verlag, 1955.

[25] P. C. Wu and J. Zhu, On the growth of solutions of the complex differential equation $f^{\prime \prime}+A f^{\prime}+B f=0$, Sci. China Ser. A 54 (2011), 939-947.

[26] S. J. Wu, On the distribution of Borel directions of entire function, Chinese Ann. Math., 14A (1993), 400-406.

[27] C. C. Yang and H. X. Yi, Uniqueness Theory of Meromorphic Functions, Kluwer Academic Publishers, New York, 2003.

[28] L. Yang, Value Distribution Theory, Springer-Verlag, Berlin, 1993.

[29] L. Yang, Deficient values and angular distibution of entire functions, Trans. Amer. Math. Soc., 308 (1988), 583-601.

[30] L. Yang and C. C. Yang, Angular distribution of $f f^{\prime}$, Sci. China Ser. A, 37 (1994), 284-294.

[31] L. Yang and G. H. Zhang, Distribution of Borel directions of entire functions, Acta Math. Sinica, 19 (1976), 157-168.

[32] G. H. Zhang, On entire function extremal for Denjoy's conjecture, Sci. Sinica (I), 24 (1981), 885-898.

[33] G. H. Zhang, Theory of Entire and Meromorphic Functions-Deficient and Asymptotic values and Singular Directions, Springer-Verlag, Berlin, 1993.

School of Mathematical Science, Guizhou Normal University, 550001, Guiyang, P.R. China.

Department of Physics and Mathematics, University of Eastern Finland, P.O. Box 111, 80101, Joensuu, Finland.

E-mail: longjianren2004@163.com 